\title{
Growth and characterization of CdMnTe by the vertical Bridgman technique
}

U. N Roy $^{1 *}$, G.S. Camarda ${ }^{1}$, Y. Cui ${ }^{1}$, G. Gu ${ }^{1}$, R. Gul ${ }^{1,2}$, A. Hossain ${ }^{1}$, G. Yang ${ }^{1}$, S.U. Egarievwe $^{1,2}$, and R.B. James ${ }^{1}$

${ }^{1}$ Brookhaven National Laboratory, Upton, NY 11973, United States

${ }^{2}$ Alabama A\&M University, Normal, AL 35762 USA

\begin{abstract}
Wegrew $\mathrm{Cd}_{1-\mathrm{x}} \mathrm{Mn}_{\mathrm{x}}$ Te crystals witha nominal Mn concentrationof 5\% by the vertical Bridgman growth technique. The structural quality of the crystal was evaluated by white beam X-ray topography in the National Synchrotron Light Source (NSLS) facility at Brookhaven National Laboratory (BNL). We observed that the crystal was free from a sub-grain boundary network, as revealed by X-ray topography and verified by our etching study. The concentration of the secondary phases, averaged over the entire ingot, was 2-3 times lower than in conventional Bridgman grown cadmium zinc telluride (CZT)crystals.
\end{abstract}

*E-mail address: uroy@bnl.gov 


\section{Introduction}

$\mathrm{Cd}_{1-x} \mathrm{Mn}_{x} \mathrm{Te}(\mathrm{CMT})$ has demonstrated goodpotential to compete with CdZnTe (CZT) [1-3], and it has several potential advantages over CZT. The firstadvantage isthat the energy band-gap of $\mathrm{Cd}_{1-}$ ${ }_{x} \mathrm{Mn}_{x}$ Teincreases about $13 \mathrm{meV}$ per atomic percent of $\mathrm{Mn}$,compared with $6.7 \mathrm{meV}$ of $\mathrm{Zn}$ in $\mathrm{Cd}_{1-}$ ${ }_{x} \mathrm{Zn}_{x} \mathrm{Te}$ [2].Thus, less manganese is needed to achieve the required band-gap compared to the addition of $\mathrm{Zn}$, so the alloy broadening effect on the transport properties should be less. The second and more important advantage of CdMnTe over CZT is that the solidus-liquidus linemerges in the wide compositional range of the pseudo-binary CdTe and MnTe system [4], thereby ensuring the near-unity segregation coefficient of $\mathrm{Mn}$ in the CdTe matrix.The segregation coefficient of $\mathrm{Mn}$ in CdTe matrix reportedly is $\sim 0.95$, which is close to unity [5], compared to 1.35 for $\mathrm{Zn}$ [6]. This near-unity segregation coefficient of Mn can ensure fairly uniform compositional homogeneity throughout the grown ingot, and ease the process of scaling up to larger ingots for lower-cost production. While at BNL, Kim et al. [7] achieved a $\mu \tau_{\mathrm{e}}$ value for $\mathrm{CMT}$ as high as $5 \times 10^{-3} \mathrm{~cm}^{2} / \mathrm{V}$. In the recent past, an energy resolution of $2.1 \%$ at $662 \mathrm{keV}$ was achieved for a $5 \times 5 \times 12 \mathrm{~mm}^{3}$ CdMnTedetector [8], proving the potential of CMT as an alternative to CZT for gamma-ray detector applications. The typical concentration of Te inclusions/precipitates observed in CMT [9] was reported as $\sim 1-3.5 \times 10^{5} \mathrm{~cm}^{-3}$, whichis similar to the values for CZT samples.However, the concentration was in the range $1-3 \times 10^{4} \mathrm{~cm}^{-3}$ for Bridgman-grown CMT, as reported by Du et al. [10]. Like CZT, the presence of sub-grain 
boundary networks, revealed by selective etching as the linear/cellular structure distribution of the etchpits, are common for CMT ingots grown by the vertical Bridgman and modified floatzone techniques [3,11]. Zhang et al.[12], on the contrary, reported sub-grain, boundary-free CMT growth using a vertical Bridgmangrowth technique. Thus, it is evident that, as far as the concentration of $\mathrm{Te}$ inclusions/precipitates and the presence of a sub-grain boundary network are concerned, theresults available in the literature are mixed. Nevertheless, the low concentration of Te inclusions/precipitations reported by Du et al [10] and the growth of CMT ingots free from sub-grain boundary networks by Zhang et al. [12] are encouraging. Evidently, CMT is worthy of further investigation in light of its potential to surpass CZT as well as resolve the somewhat differing observations.

In this present work, we grew CMT ingots by the vertical Bridgman technique and characterized them for the concentration and size distribution of Te inclusions/precipitates along the ingot's radial and axial directions. Emphasis was placed on investigating the presence of a sub-grain boundary network/cellular structure of dislocation walls by X-ray topography and selective chemical-etching studies.

\section{Experimental}

We grew $\mathrm{Cd}_{0.95} \mathrm{Mn}_{0.05}$ Teingots by the vertical Bridgman growth technique. The crystals were grown in conically-tipped quartz ampoules, and the inner wall was coated with graphite by cracking spectroscopic-grade acetone at $900{ }^{0} \mathrm{C}$. The coated ampoules then were annealed under a high vacuum at about $1100{ }^{\circ} \mathrm{C}$, followed by slow cooling down to room temperature.

Stoichiometric amounts of the raw materials then were loaded into the ampoules and sealed under a dynamic vacuum of $2 \times 10^{-6}$ torr. The starting materials used for growing CMT were asreceived 6N purity CdTe from 5N Plus Inc., 6N purity Te, and 99.9998\% pure Mn from Alfa 
Aesar. To minimize the incorporation of impurities during handling, synthesis and growth were carried out in the same ampoule. The ingots were grown in a three-zone vertical Bridgman furnace at a rate of $\sim 2 \mathrm{~cm} /$ day under a temperature gradient of $7-10{ }^{0} \mathrm{C} / \mathrm{cm}$ near the melt/solid interface. The ingot was cooled to room temperature at a rate of $8^{0} \mathrm{C} / \mathrm{hr}$ after growth was completed.

The grown ingots then were removed from the ampoule. They slid out easily without sticking to the ampoule wall. The whole ingot was cut into wafers perpendicular to its axis using a diamond-impregnated wire saw, in order to observe the grain growth and study the Te inclusions/precipitates along the ingot's axis. The wafers were lapped with SiC lapping paper with different grit sizes, and finally polished to a mirror-like finish using a $0.05-\mu \mathrm{m}$ alumina suspension on a felt pad. The concentration and size distribution of Te inclusions/precipitates in the polished wafers taken from different positions along the ingot's radial and axial directions were evaluated by an automated IR-transmission microscopy system. Studies of the crystalline quality and presence of extended defects were carried out by White Beam X-Ray Diffraction Topography (WBXDT) in the reflection mode using the X19C beam line at BNL's NSLS facility. The grains and etch pits were revealed by a Saucedo chemical-etching solution [13].

\section{Results and discussion}

Figure 1 is an optical photograph of an as-grown $\mathrm{Cd}_{0.95} \mathrm{Mn}_{0.05}$ Te ingot grown by the vertical Bridgman technique. The ingot is $22 \mathrm{~mm}$ in diameter and $10.5 \mathrm{~cm}$ in length. As is evident from the figure, the upper half contains many twins. CMT is known to be prone to twins $[10,12]$ due to the low stacking-fault energy, and the presence of twins in our ingot agrees with prior observations. The wafers cut perpendicular to the ingot's axis from the tip to the top of the ingot are shown in Figure 2. To reveal the grain boundaries and twins, all the wafers were polished to a 
mirror-like finish followed by selective etching using a Saucedo solution [13]; the picture of the face of the tip of the ingot is shown in the inset indicated by the arrow. The ingot is large-grain polycrystalline in nature, as is evident from the Fig.2. It is apparent that grain 2 increases up to the middle of the ingot's length, while, after that, the growth of grain 1 dominates. Few twins are apparent and are found to multiply from the middle of the ingot towards the top of the ingot, as shown in Fig. 2.Extensive investigations on the size distribution and concentration of the secondary phases(Te inclusions/precipitates)were carried out along the diameter of the ingot as well as along its length. The cross-sectional 5-mm-thick wafers taken from different distancesalongthe ingot were polished on both sides to a mirror-like finish for an infrared (IR) transmission microscopy study for quantifying the size and distribution of secondary phases. Figures 3 and 4 show the etched, polished, and corresponding IR transmission pictures of the full wafers taken from $\sim 2 \mathrm{~cm}$ and $\sim 9.5 \mathrm{~cm}$ from the tip of the ingot. The same wafers are indicated by white circles in Fig.2. As shown in Fig. 3c, the grain boundaries are highly decorated by secondary phases, as indicated by the arrows. Figures $5 \mathrm{a}$ and $\mathrm{b}$ show high-magnification microscopy pictures of the grain boundary in the reflection mode and the corresponding IR transmission mode, respectively. No decoration by secondary phases (inclusions or precipitates) was observed at the twin planes indicated by the ellipse in Figs. $3 b$ and 3c. These are likely coherent twin boundaries; such boundaries are low-energy interfacial planes [14]. Highmagnification microscopy pictures of the coherent twin lamella in the reflection and IR transmission modes are shown in Figures $6 \mathrm{a}$ and 6b, respectively, depicting no secondary phases at the twin boundaries.Meanwhile, the twins indicated in Fig.4a are found to be decorated with secondary phases(Fig.4c). These twins perhaps are incoherent twin planes and possess high interfacial energy relative to the coherent twin plans. The incoherent twin planes decorated with 
secondary phases have an adverse effect on the detector's performance ${ }^{9}$ and generally are excluded during the detector's fabrication, which is also the case for high-angle grain boundaries. The secondary phaseswere distributed randomly over the full area of the wafers (Figs. 3c and 4c). The size distribution and concentrations of the secondary phases were evaluated along the diameter of the wafers taken from different lengths of the ingot. Figure 7 shows variationsin the concentrations of the secondary phases along the diameter of the wafers from different distances from the tip of the ingot. All the wafers investigated for the measurementwere 5-mm thick after polishing. The concentrations along the diameter of the wafers are fairly random, as is also evident from Figures $3 \mathrm{c}$ and $4 \mathrm{c}$. Figure 8 plots the total concentrations of Teinclusions/precipitates averaged over the diameter of each wafer along the ingot's length. The average concentrationsof the Teinclusions/precipitates vary from $\sim 1 \times 10^{5} \mathrm{~cm}^{-3}$ to $\sim 1.8 \times 10^{5} \mathrm{~cm}^{-3}$ from the tip to the top of the ingot. These concentration values agree well with those reported by Babalola et al.[9]. The reported values are $9.5 \times 10^{4} \mathrm{~cm}^{-3}$ and $3.5 \times 10^{5} \mathrm{~cm}^{-3}$ for two different ingotsgrown by the Bridgman technique [9]. The concentration of Teinclusions/precipitates increased very slightly from the tip to $\sim 7.5 \mathrm{~cm}$ from it, while the concentration was observed to increase at a higher rate for wafers taken from the rest of the ingot towards its top. According to the definition of Rudolph [15],thosesecondary phases larger than $1 \mu \mathrm{m}$ can be considered as Te inclusions. This increase in Te inclusions along the growth direction also was reported by Rudolph [15], and the results are in good agreement with our observations. This increase along the direction of growth might reflect the enrichment of the melt with Te during the growth process, and the larger amount of Te captured at the growth interface [15]. A typical IR transmission image of the Te inclusions is shown in Figure 9a; magnified views of the shape of large Te inclusions are shown in Figs. 9b and 9c. The histograms of the 
size distribution and concentrations of the secondary phasesat the lowest and highest points,as indicated by the circles in Fig.7, are shown in Figs. 10a and 10b. In general, the histogram extends up to $\sim 20 \mu \mathrm{m}$ in size, similar to that for the CdZnTe matrix [16]. However, according to the values in the literature and the present observations, the total average concentration of the secondary phasesis less than the values for CdZnTe ingots grown by the Bridgman technique. In many regions of the ingot, we observed occasional occurrences of larger diameter Te inclusions (30-40 $\mu \mathrm{m})$ (the histograms are not shown here) by high magnification IRtransmission microscopy, as illustrated in Figs. $9 b$ and $9 c$.

The structural quality of the CMT crystals was characterized using SynchrotronWhiteBeamXrayDiffractionTopography (WBXDT) inthe reflection mode. Care was taken to minimize the stress during polishing and handling. The wafer was polished on both sides to a mirror-like finish using a South Bay Technology Model \#920 polishing machine. The polished wafer then was chemo-mechanically polished on a felt pad with a bromine methanol-ethylene glycol mixture, followed by chemical etching in a $2 \%$ bromine methanol solution for two minutes to remove any damaged layer after mechanical polishing. The etched wafer was used to illustrate the grains; the X-ray topographic image of the corresponding grains is indicated by the arrows shown in Figure 11. The streakiness of the topographic image near the periphery of the wafer, denoted by the small arrows, is an indication of lattice distortion; it suggests that the ampoule wall is responsible for introducing stress either during growth or the post-growth cooling process. The occurrence of strain near the vicinity of the ampoule wall is very common for CdTe ingots grown by the Bridgman method or via physical vapor-transport growth[17,18].These strain fields can extend over the entire ingot [18]. However, in the present case, the streaks near the periphery of the wafer are localized as shown by the small arrows in Fig. 11. A similar streaky pattern, and hence, 
lattice distortion also was observed via x-ray topography measurements around the periphery of contactless CdZnTe wafers grown via multi-tube physical-vapor transport [19].However, in a similar study for $\mathrm{CdTe}_{\mathrm{x}} \mathrm{Se}_{1-\mathrm{x}}$,no such lattice distortion was observed near the periphery of the wafers grown by either the Bridgman method or the Traveling Heater method [20,21], and the wafers were nearly stress free. Very high lattice distortion, and hence large stress is evident near the grain boundary marked inside the circle in Fig.11. The occurrence of large stress possibly is caused by the pointed nature of the grain boundary shown by the blue arrows. All the thermal stress perhaps concentrated at the pointed tip, leaving behind the large lattice distortion near the grain boundary. No such stress was observed at the reasonably straight grain boundary, as indicated by the arrows in Fig.11, indicating the uniform relaxation of the stress along the boundary. The twins near the left-hand side of the wafer are distorted in the topographic image, possibly due to the stress developed from interactions with the ampoule's wall. In general, except for these regions, the wafer is fairly stress-free, and no sub-grain boundary network was evident from the topographic picture. However, a few sub-grain boundaries are present in the wafer as dark and whitelines, as illustrated in Fig.11. The white lines correspond to the separation of the diffracted images of the adjacent sub-grains, while the dark lines correspond to the overlapping of the diffracted images. The relatively low thermal-gradient during the CMT growth possibly is the reason for the nearly stress-free and free from subgrain boundary network. Zhang et al. [12] also reported a sub-grain boundary network free CMT grown by the vertical Bridgman growth technique under a thermal gradient of $13 \mathrm{~K} / \mathrm{cm}$. No cellular etch pits, which are the signature of a sub-grain boundary network, were observed by their etching study [12]. However, a high concentration of sub-grain boundary networks was observed for CMT ingots grown by the vertical Bridgman technique under a thermal gradient of $50{ }^{\circ} \mathrm{C} / \mathrm{cm}$ [3]. CMT ingots, grown by a 
modified float-zone technique using an optical furnace, also showed a heavily decorated subgrain boundary network as revealed by theiretching study [11]. Our X-ray topographic study of an optically heated modified float-zone grown CMT revealed a high stress field, and thus, high lattice distortion throughout the wafer [22]. Its presence possibly was due to the very high temperature gradient imposed by the optical float-zone technique, whichresults in very high thermal stress in the grown ingots. Such stresses are responsible for the large lattice distortion and the formation of a sub-grain boundary network because of the poor thermal conductivity of CdTe-based materials. Thus, growth in a low thermal gradient, possibly is favorable for CdTebased materials in order to minimize the thermal stress and formation of sub-grain boundary networks. In our present study, the thermal gradient used was $7-10{ }^{0} \mathrm{C} / \mathrm{cm}$. In agreement with our X-ray topographic data, nosub-grain boundary network was revealed by our chemical-etching study. Figure 12 shows the typical distribution of etch pits in our CMT wafers. No cellular distribution of etch pits was observed, as illustrated in Fig.12.

\section{Summary}

We grew CdMnTe crystals by the vertical Bridgman growth technique, andinvestigated the defect structure and crystalline quality of the grown ingots. The radial-and axial- concentration and size distribution of the secondary phases also were explored. The average concentration of the secondary phases was observed to be lower than that in CZT grown using the vertical Bridgman method. Our X-ray topographic analyses and etch-pit study evidently showed that the crystal is free from sub-grain boundary networks and fairly stress-free, possibly due to the low thermal gradient during growth. However, the resistivity of our grown ingot is lowerthan the required value for realizing detector-grade material. We are making efforts to increase their resistivity by optimizing the In concentration. 


\section{Acknowledgments}

This workhasbeensupportedbythe

U.S.DepartmentofHomelandSecurity,DomesticNuclearDetectionOffice, undercompetitively awardedcontract/IAAawardnumber 2012-DN-077-ARI065-03.Alabama

A\&MUniversityresearcherswerealsosupportedbytheU.S.

NuclearRegulatoryCommissionthroughawardnumberNRC-27-10-

514.BNLscientistsreceivedsupportfromtheU.S.DepartmentofEnergy'sOffice

ofDefenseNuclearNonproliferationResearchand Development,NA-22.This

supportdoesnotconstituteanexpressed orimpliedendorsementbytheU.S.Government.The

manuscript has been authored by Brookhaven Science Associates, LLC under Contract No. DEAC02-98CH1-886 with the U.S.

\section{References:}

1. A. Burger, K. Chattopadhyay, H. Chen, J.O. Ndap, X. Ma, S. Trivedi, S.W. Kutcher, R. Chen, and R.D. Rosemeier, J. Cryst. Growth 198/199 (1999) 872.

2. A. Mycielski, A. Burger, M. Sowinska, M. Groza, A. Szadkowski, P. Wojnar, B. Witkowska, W. Kaliszek, P. Siffert, Phys. Status Solidi C2 (2005) 1578.

3. A.Hossain,Y.Cui,A.E.Bolotnikov,G.S.Camarda,G.Yang,D.Kochanowska, M. Witkowska-Baran,A.Mycielski,R.B.James,J. Electronic Materials38(2009)1593.

4. R. Triboulet, A. Heurtel and J. Rioux, J.Cryst. Growth 101 (1990) 131.

5. J. Zhang, W. Jie, T. Wang, D. Zeng, Y. Hao, and K. He, J. Cryst. Growth 310 (2008) 3203.

6. A. Tanaka, Y. Masa, S. Seto, and T. Kawasaki, J. Cryst. Growth 94 (1989) 166.

7. K.H. Kim, G. Jeng, P. Kim, J. Choi, A. E. Bolotnikov,G. S. Camarda, and R. B. James, J. Appl. Phys. 114(2013) 063706. 
8. K.H. Kim, A. E. Bolotnikov, G. S. Camarda, R. Tappero, A. Hossain, Y. Cui, J. Franc, L. Marchini, A. Zappettini, P. Fochuk, G. Yang, R. Gul, and R. B. James, IEEE Trans. Nucl. Sci. 59 (2012) 1510.

9. O.S. Babalola, A.E. Bolotnikov, M. Groza, A. Hossain, S. Egarievwe, R.B. James, A. Burger, J. Cryst. Growth 311 (2009) 3702.

10. Y. Du, W. Jie,T. Wang,Y. Xu,L. Yin,P. Yu, and G. Zha, J. Cryst. Growth 318 (2011) 1062.

11. A. Hossain, G.D. Gu, A.E. Bolotnikov, G.S. Camarda, Y. Cui, U.N. Roy, G. Yang, T. Liu, R. Zhong, J. Schneeloch, and R.B. James, Nucl. Instrum. Methods A 784 (2015) 33.

12. J. Zhang,W. Jie, T. Wang, D. Zeng, Y. Hao, and K. He, J. Cryst. Growth 310 (2008) 3203.

13. E. Saucedo, P. Rudolph and E. Dieguez, J. Cryst. Growth 310 (2008) 2067.

14. J. Zhang, W. Jie, L. Wang, and L. Luan, Cryst. Res. Technol. 45 (2010) 7.

15. P. Rudolph, Prog. Crystal Growth and Charact. 29 (1994) 275.

16. A.E. Bolotnikov, S. Babalola, G.S. Camarda, Y. Cui, S.U. Egarievwe, R. Hawrami, A. Hossain, G. Yang, and R.B. James,IEEE Trans. Nucl. Sci. 57 (2010) 910.

17. C-H Su, S.L. Lehoczky, B. Raghothamachar, and M. Dudley, Mat. Sci. Eng. B 147 (2008) 35 .

18. W. Palosz, K. Grasza, K. Durose, D.P. Halliday, N.M. Boyall, M. Dudley, B. Raghothamachar, and L. Cai, J. Cryst. Growth 254 (2003) 316.

19. C.K. Egan, A. Choubey, M. Moore, and R.J. Cernik, J. Cryst. Growth 343 (2012) 1.

20. U.N.Roy, A.E. Bolotnikov, G.S. Camarda, Y. Cui, A. Hossain, K. Lee, W. Lee, R. Tappero, G. Yang, Y. Cui, A. Burger, R.B.James, J. Cryst. Growth 411 (2015) 34. 
21. U.N. Roy, A.E.Bolotnikov,G.S.Camarda,Y.Cui,A.Hossain,K.Lee,G.Yang, and R.B.James, J. Cryst. Growth 389 (2014) 99.

22. G. Yang, G. Gu, A.E. Bolotnikov, Y. Cui, G.S. Camarda, A. Hossain, U.N. Roy, N. Kivi, T. Liu, and R.B. James, Electron. Materials Lett. 11 (2015) 500.

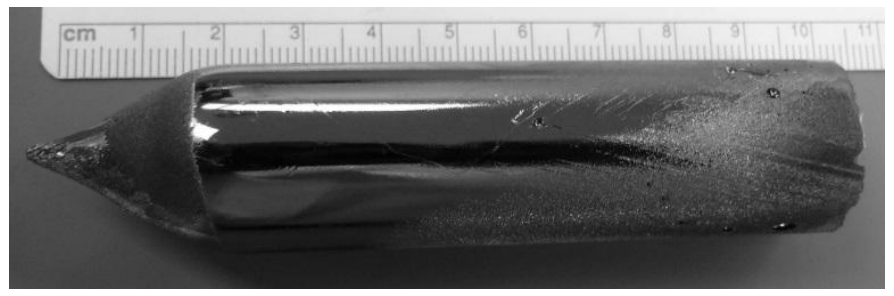

Figure 1.Optical photograph of the as-grown CMT ingot grown by the vertical Bridgman technique. 


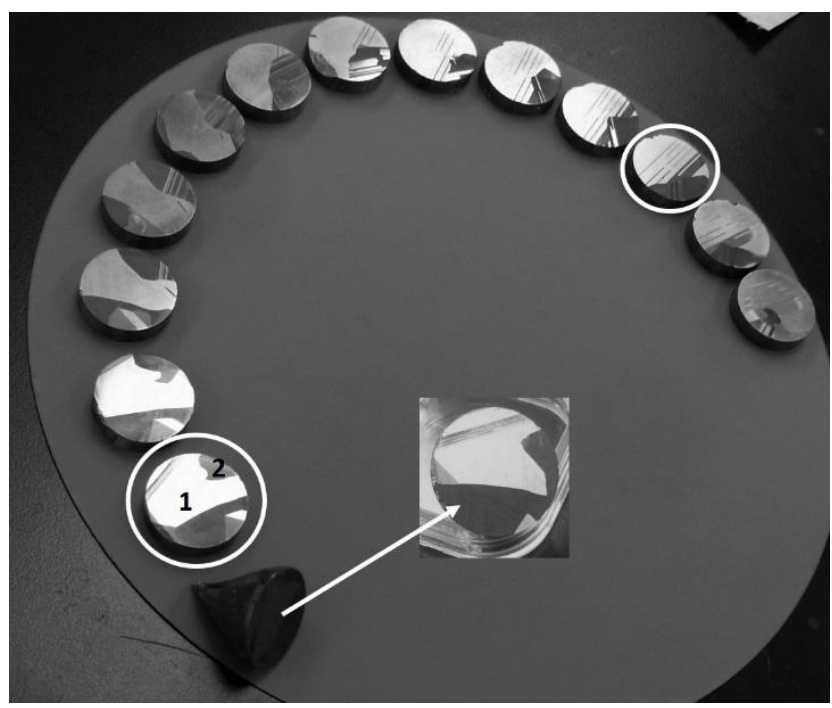

Figure 2. Optical photograph of the etched wafers cut perpendicular to the ingot axis at different distances from the tip.

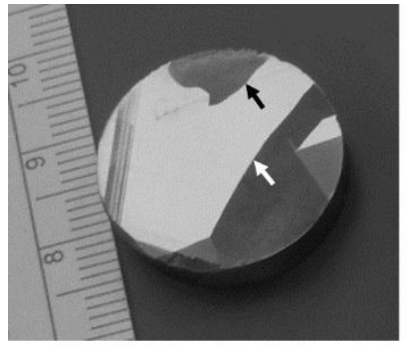

a

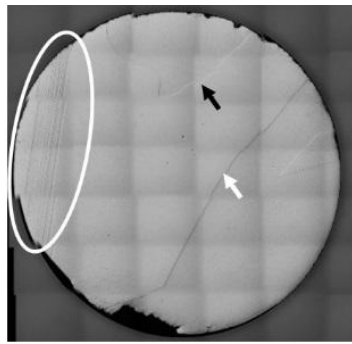

b

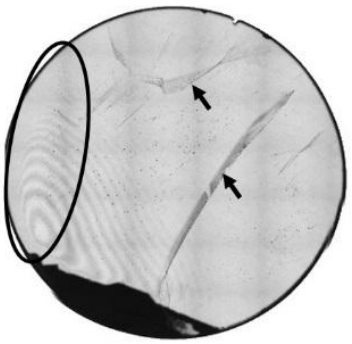

c

Figure 3. Wafer cut perpendicular to the ingot axis $\sim 2 \mathrm{~cm}$ from tip: a) etched, b) polished, and c) IR transmission picture of the full wafer. The coherent twins are denoted by the ellipse, and the corresponding IR transmission image shows no secondary phases at the twin boundaries. The grain boundaries are marked by the arrows, which are highly decorated with secondary phases. 


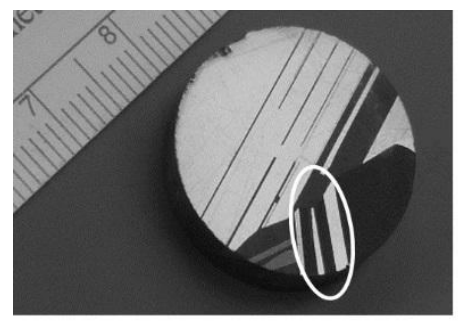

a

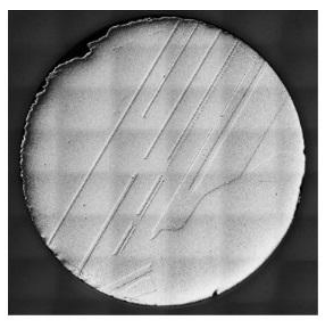

b

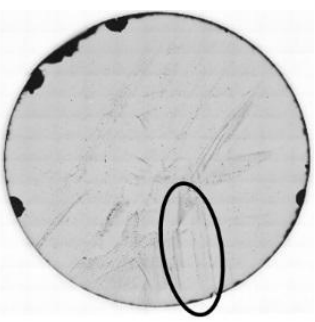

c

Figure 4. Wafer cut perpendicular to the ingot axis $\sim 9.5 \mathrm{~cm}$ from tip: a) etched, b) polished, and c) IR transmission picture of the full wafer. The incoherent twins are denoted by the ellipse, and the corresponding IR transmission image shows the presence of secondary phases at the twin boundaries.

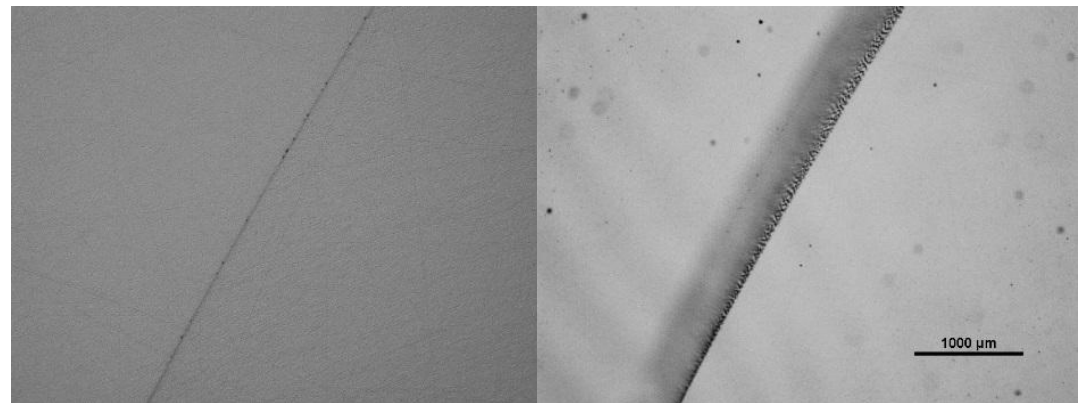

a)

b)

Figure 5. a) Optical micrograph of a grain boundary in the reflection mode, and b) the corresponding region shown in an IR transmission mode.

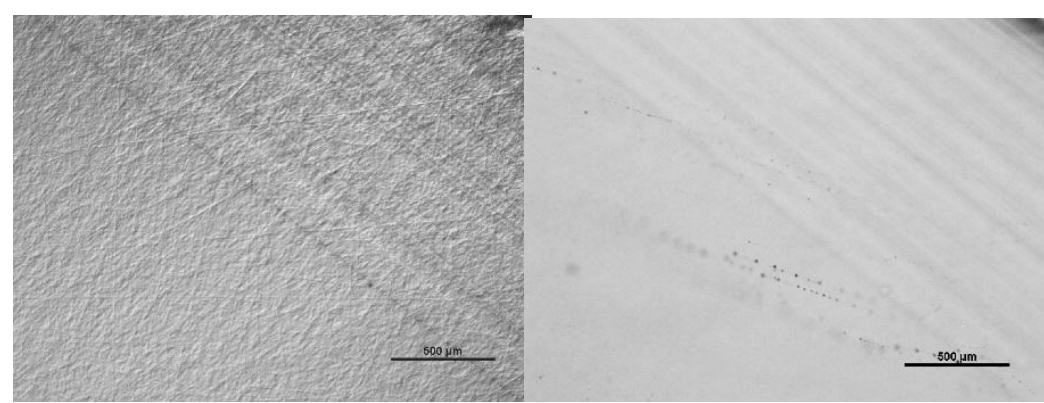

a)

b)

Figure 6. a) Optical micrograph of twin lamellae in a reflection mode, and b) the corresponding region in an IR transmission mode. 


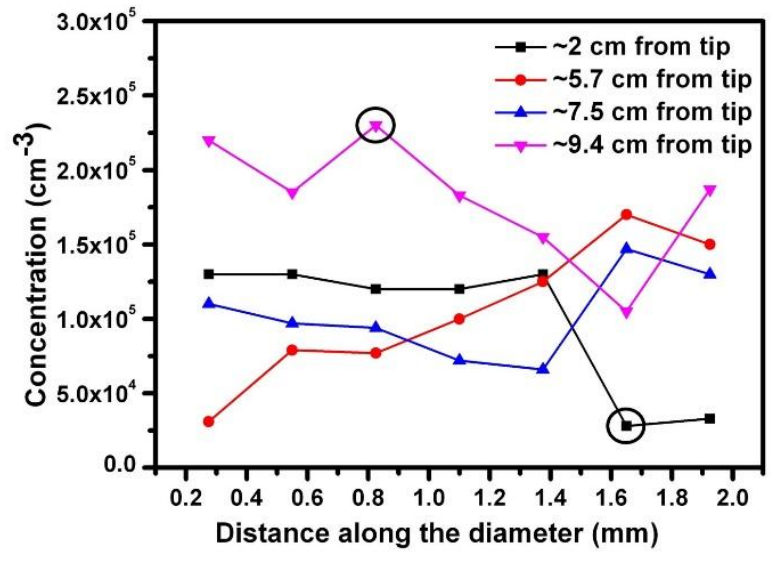

Figure 7.Distribution of Te inclusions/precipitates along the diameter of the wafers taken from wafers cut from different locations along the axis of the ingot.

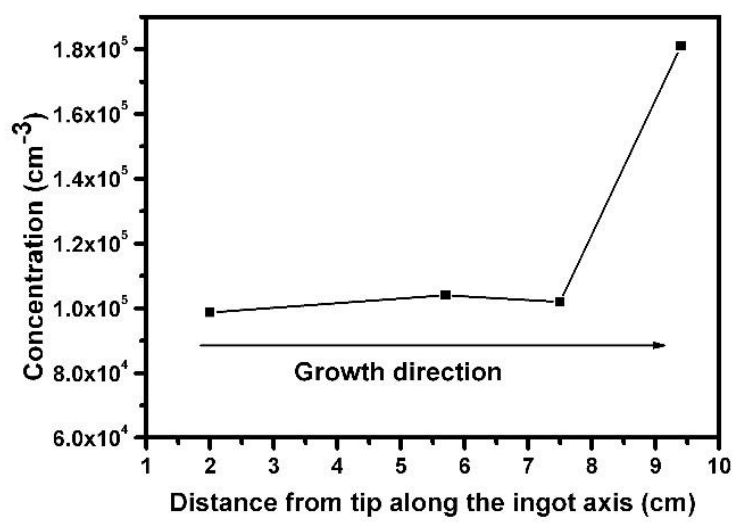

Figure 8. Distribution of Te inclusions/precipitates along the length of the ingot. (Each point is the average value of the concentration over the diameter of the wafer). 


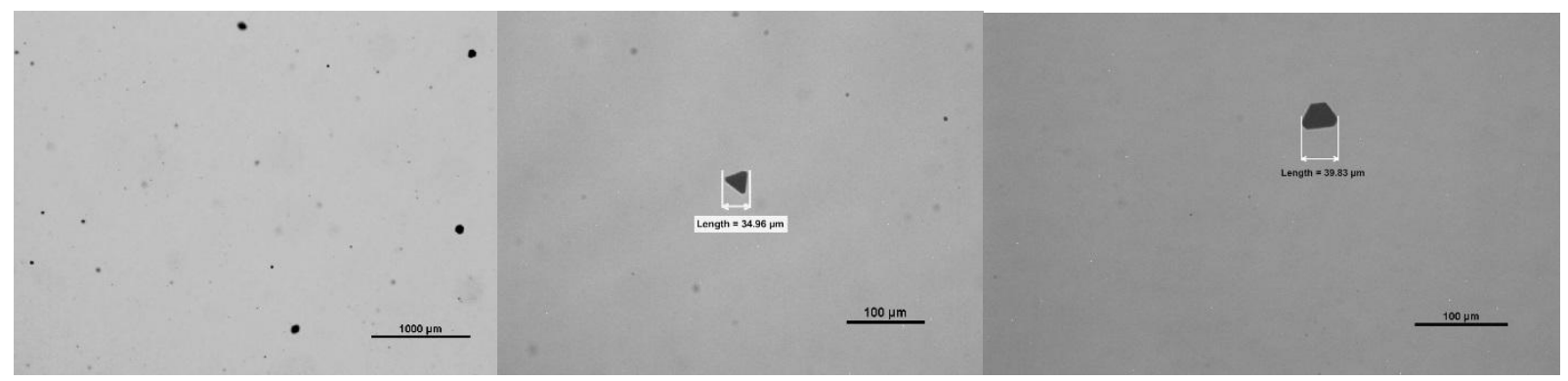

a) b)

c)

Figure 9. a) Typical IR transmission microscopy image showing the Te inclusions/precipitates; b) and c) are high magnification IR transmission imagesshowing the morphology of the Te inclusions. The size of the inclusions are indicated in the pictures.

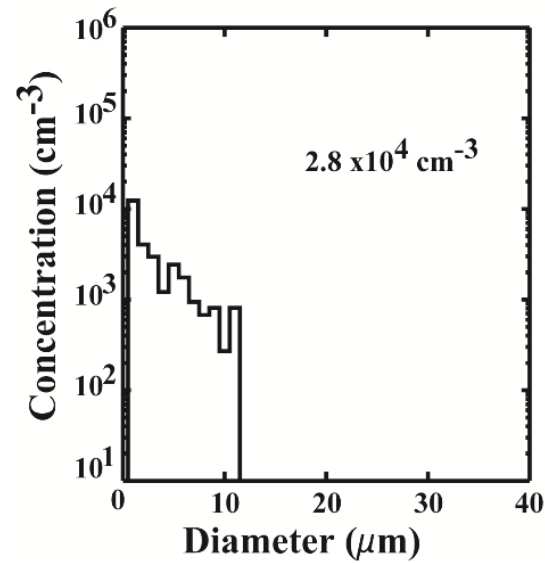

a)

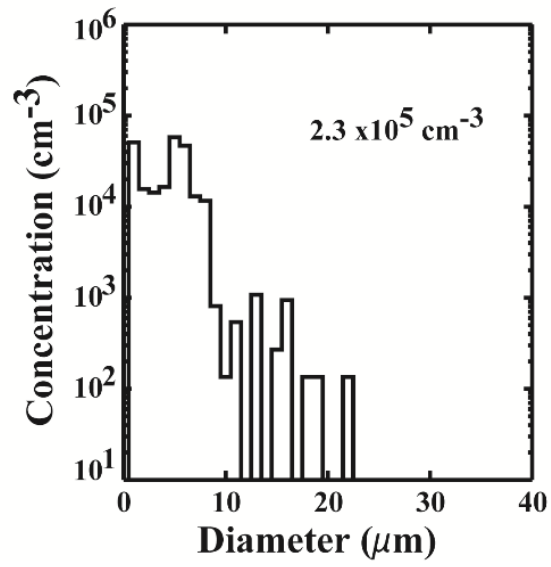

b)

Figure 10. Concentration and size distribution of Te inclusions/precipitates in as-grown CMT sample: a) lowest and b) highest concentration region indicated by the circle in Fig.7. 


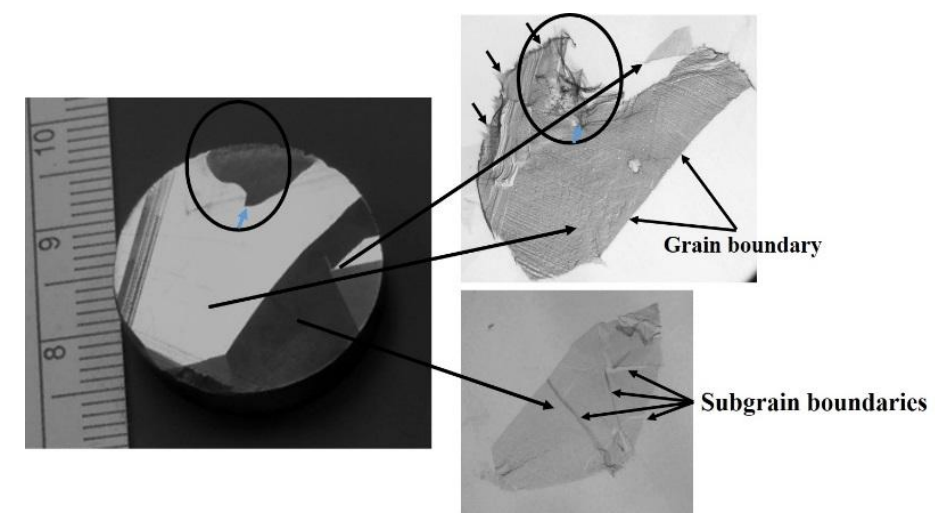

Figure 11. Optical picture of the etched wafer and the X-ray topographic image of the corresponding grains. A high lattice-distortion region is circled in the photo, and the grain boundary is indicated by the blue arrow.The sub-grain boundaries and the grain boundaries are donated by the arrows. The small arrows on the left upper corner indicate interactions of the ingot with the ampoule wall.

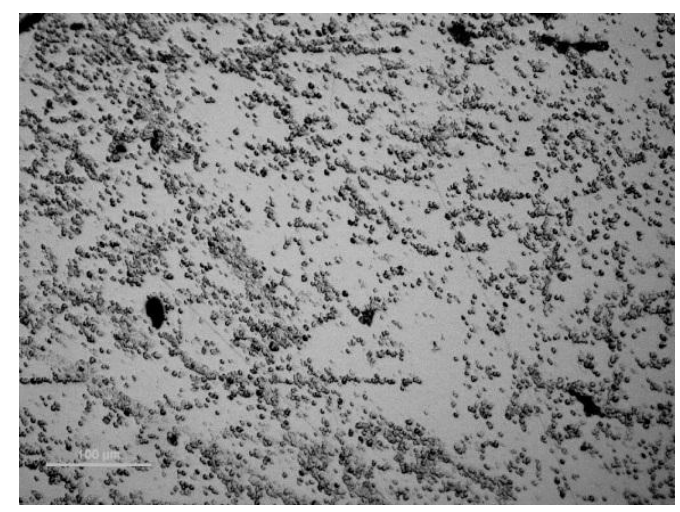

Figure 12. Optical micrograph of the chemically etched surface of anas-grown CMT sample. 